\title{
Fachkommunikation
}

\author{
Karin Luttermann*
}

\section{Klare Sprache als Mittel für Fachkommunikation und Wissenstransfer}

https://doi.org/10.1515/iwp-2017-0043

Zusammenfassung: In der linguistischen Pragmatik wird der Ausdruck Kommunikation eingesetzt, um Zwecke der Verwendung von Sprache und Funktionen menschlichen Verständigungshandelns zu erfassen. Der Kommunikationsbegriff erstreckt sich auf diverse Domänen und Situationen der Kommunikation (Alltag, Beruf, Fach) und umfasst verschiedene Kommunikationskonstellationen und Adressaten (Experten, Laien, Behinderte, Migranten, Deutschlernende). Für die Kommunikation mit Menschen, die eine Behinderung haben, wird auf die Leichte Sprache als Kommunikationsmittel zurückgegriffen. Die Leichte Sprache ist aus praxisorientiertem Handeln entstanden; die (Sprach-) Wissenschaft steht weithin noch außen vor. Erst allmählich scheint die Linguistik sich dem Forschungsgegenstand Leichte Sprache zuzuwenden. Der vorliegende Beitrag plädiert - sprachwissenschaftlich fundiert - für den Gebrauch der „klaren Sprache“ und für eine Perspektivenerweiterung des Laienbegriffs innerhalb der Experten-Laien-Kommunikation. Hier steht eine theoretisch, empirisch und angewandt begründete Forschung erst am Anfang.

Deskriptoren: Kommunikation, Sprache, Sprachbarriere, Recht, Forschung

\section{Clear language as a means of language for special purposes and knowledge transfer}

Abstract: In linguistic pragmatics the expression communication stands for analyzing the purposes of the use of language and the functions of linguistic actions for human understanding. The concept of communication covers various domains and situations (everyday life, profession) and includes different constellations of communication and addressees (experts, lay people, disabled people, migrants, learners of the German language). For communicating with people with disabilities, plain language is used as a means of communication. But plain language has

*Kontaktperson: Prof. Dr. Karin Luttermann, Katholische Universität Eichstätt-Ingolstadt, Sprach- und Literaturwissenschaftliche Fakultät, E-Mail: karin.luttermann@ku.de emerged from practice. (Linguistic) science has hardly been involved. It is only slowly that linguistics is applying itself to this subject. On a sound linguistic basis, the present article argues for the use of the term "clear language" and for differentiating the perspective of the non-experts within expert-lay communication. Here, a theoretical, empirical and applied research is only in its initial stages.

Descriptors: Communication, Language, Language barrier, Justice, Research

\section{La langue claire comme moyen de communication professionnelle et de transfer des savoirs}

Résumé: En pragmatique linguistique, le terme « communication » fait référence à l'usage du langage et des fonctions de l'action linguistique dans les interactions humaines. Le concept de la communication s'étend à divers domaines et situations (vie quotidienne, contexte professionnel) et il recouvre aussi des situations d'interaction et des locuteurs très variés (experts, non-experts, personnes handicapées, migrants, personnes apprenant l'allemand). Pour communiquer avec des personnes en situation de handicap mental, on a souvent recours à une langue dite " simplifiée » comme moyen de communication. Cette langue «simplifiée » est une langue, née de l'usage pratique quotidien. La linguistique ne s'intéresse que depuis peu à la «langue simplifiée » comme objet d'étude. Sur base de recherches linguistiques, cet article plaide pour l'utilisation de l'expression « langue claire » et pour un élargissement du sens du terme «non-expert » en désignant la communication entre experts et non-experts. La recherche théorique, empirique et appliquée dans ce nouveau domaine n'en est qu'à ses débuts.

Descripteurs: Communication, Usage du langage, Barrière de la langue, Droit, Recherche

\section{Einleitung}

Das Wort „Kommunikation“ bedeutet Teilhabe. Das Substantiv Kommunikation stammt von lt. communicatio, was 
so viel wie $>$ Mitteilung $<$ und $>$ gemeinschaftlich tun $<$ (zu lt. con $>$ gemeinsam $<$ und lt. munus $>$ Aufgabe, Leistung $<$ ) bedeutet. In der linguistischen Pragmatik wird der Ausdruck „Kommunikation“ eingesetzt, um Zwecke der Verwendung von Sprache und Funktionen menschlichen Verständigungshandelns in bestimmten Kommunikationsbereichen $\mathrm{zu}$ erfassen. Kommunikation ist weiter gefasst als Sprache und umfasst neben sprachlichem Handeln paralinguistisches, non-verbales und interaktionales Handeln. Die Ebene der Interaktion betrifft die Verständigung zwischen Textproduzent und Rezipient.

Das linguistische Konzept der kommunikativen Verständigung ist in der Pragmatik kein technischer Übertragungsprozess von Input und Output (vgl. Shannon/ Weaver 1949). Vielmehr zeichnet sich die menschliche Kommunikation dadurch aus, dass Information durch Zeichen (Sprachzeichen, Bilder, Piktogramme) ausgedrückt wird und die Kommunizierenden semantische Bedeutung aushandeln. Verständigung ist also im Sinne von Verständlichkeit und Verstehen zu begreifen. Dabei ist wichtig zu betonen, dass der Rezipient auf der Grundlage seines aktuellen Wissensstandes auf das Kommunikationsangebot reagiert und einen Handlungsprozess initiiert. Das Ziel ist ein übereinkommendes Sich-Verstehen.

Der Beitrag erörtert handlungspragmatisch die Frage, welche Bedeutung die klare Sprache als Kommunikationsmittel in der externen Fachkommunikation hat. Zuerst werden Textlinguistik und Experten-Laien-Kommunikation (Kap. II, III) in den Kontext der Leichten Sprache gestellt. Dann thematisiere ich den Adressatenkreis und die Entwicklung der Leichten Sprache und erkläre kommunikativfunktional meinen Begriff der klaren Sprache (Kap. IV, V, VII). Das Gebot der Klarheit wird mit dem Rechtslinguistischen Verständlichkeitsmodell für die Rechts- und Verwaltungskommunikation operationalisiert (Kap. VI, VIII, IX).

\section{Kommunikationsorientierte Textlinguistik}

Die kommunikationsorientierte Textlinguistik blickt vom Text auf den Satz und wendet sich spezifischen Sprachgebrauchsphänomen zu (vgl. Hartmann 1971: 25). Sie behandelt Texte als „Äußerungen-in-Funktion“ (Adamzik 2016: 31), denen im Vergleich zu sprachrichtigen Sätzen komplexe Regeln zugrunde liegen. Danach hat der Satz einen nur instrumentalen Charakter als Textbildungsmittel. Ihm übergeordnet sind die Textfunktion und der Handlungskontext, der durch die Handlungsabsicht des Emittenten und durch außersprachliche Faktoren wie die Art der Partnerbeziehung (Rollenverhältnis, Grad der Bekanntschaft) oder Annahmen über Vorwissen und Werte der Textrezipienten geprägt ist. Textsorten sind „konventionell geltende Muster für komplexe sprachliche Handlungen“ (Brinker 2005: 144), die für die Textproduktion und Rezeption eine Orientierung geben.

Klaus Brinker (2005: 112ff.) unterscheidet fünf textuelle Grundfunktionen (Information, Appell, Obligation, Kontakt, Deklaration). Ein Informationstext dient dem Textproduzenten dazu, dem Rezipienten zu sagen, dass er ihn über etwas Bestimmtes informieren will. Mit einem Appelltext gibt er ihm zu verstehen, dass er ihn dazu bewegen will, eine bestimmte Handlung zu vollziehen. Die Funktionen bezeichnen die Handlungsabsicht des Produzenten, als was der Adressat den Text auffassen soll. Der Schreiber versucht, mit dem Text in einer bestimmten Weise auf den Leser einzuwirken. Das Einwirkenwollen ist zielgerichtetes, intentionales Handeln. Gelingende Kommunikation bedingt, dass ein Text für seine Adressaten verständlich ist. Das Verständlichkeitsgebot gilt besonders für juristisch-administrative Texte, die das Alltagsleben tangieren. Zweck von Gesetzen ist, Verhaltensregeln, die Rechte und Pflichten begründen, $\mathrm{zu}$ formulieren und durchzusetzen.

\section{Experten-Laien-Kommunikation}

Wissenschaftlich betrachtet ist die Frage nach der Verständlichkeit von Texten eng mit der Fachsprachenforschung verbunden. Fachsprachen gelten als Mittel für eine optimale Verständigung über ein Fachgebiet unter Fachleuten. Von der interfachlichen Kommunikation ist die fachexterne Kommunikation zu unterschieden, wo der Experte eines bestimmten Faches mit einem Laien interagiert. In der Interaktion kann es zu - teils erheblichen Kommunikationsstörungen kommen, wenn die Fachsprache in den gesellschaftlichen Diskurs einfließt, ohne dass dabei das Laienverständnis adäquat berücksichtigt wird. Belege dafür liefert die empirische Verständlichkeitsforschung insbesondere in den Kommunikationsbereichen Medizin, Recht und Verwaltung. Für die Rechtskommunikation ist konstitutiv, dass die Gesetzessprache auf die Gemeinsprache zurückgreift, um Lebenssachverhalte zu regeln. Gemeinsprachliche Ausdrücke werden im Rechtskontext aber anders konzeptualisiert. Die Konzepte bilden für die Rechtsexperten die Grundlage ihrer kategorialen Wahrnehmung.

Beim Übergang von der Alltags- in die Gesetzessemantik kommt es zu einer Spezifikation, die Laien nicht ohne weiteres erkennen. Man leiht sich zum Beispiel ein Auto 
vom Händler, ein Buch vom Kollegen oder Eier vom Nachbarn. In der Gemeinsprache kann die Leihe also zur Bezeichnung mehrerer Nutzungsarten (wie mieten, borgen, ausleihen, verleihen) verwendet werden. Nicht so im Gesetz. Juristen meinen mit Leihe eine unentgeltliche Gebrauchsüberlassung und Rückgabe einer Sache (§598 BGB). Danach wird nur das Buch verliehen. Bei Eiern handelt es sich um ein Darlehen (§ 607 BGB), da sie nach dem Verzehr nicht in gleicher Güte zurückgegeben werden können. Der Leihwagen ist ein Mietwagen. Man mietet ihn und zahlt für die Abnutzung einen Mietzins ( $\$ 535$ BGB). Das Beispiel steht stellvertretend für seine Art (vgl. Luttermann 2010 a: 141 f.; 2010 b: 147; 2015: 281 ff.; 2016: 177 ff.).

Eine wesentliche Aufgabe, die sich bei der Weiterentwicklung der Experten-Laien-Kommunikation stellt, ist, das Laienkontinuum (vom Durchschnittsbürger bis hin zu Leichte Sprache-Lesern) auszudifferenzieren und in den fachlichen Diskurs nachhaltig einzubinden. Das Laienwissen ist graduell und fließend. Anhand der W-Fragen lassen sich relevante Handlungsbedingungen der Kommunikationssituation bestimmen: Wer kommuniziert wie wann wo mit wem zu welchem Zweck? Die Fragen bilden zugleich die Grundlage für Textumformungen ${ }^{1}$ in klare Sprache und auf unterschiedlichen Niveaustufen (Kap. VII.3). - Wer sind die anvisierten Adressaten von Leichter Sprache?

\section{Adressaten der Leichten Sprache}

\section{Grundlagen}

Leichte Sprache steht in dem Bemühen, Kommunikationsbarrieren abzubauen, damit möglichst viele Menschen am Zusammenleben in einer Gesellschaft teilhaben können. Kommunikation ist ein konstitutives Merkmal menschlichen Handelns. Eine Barriere, die die Kommunikation betrifft, erschwert oder macht es sogar unmöglich, dass bestimmte Personengruppen $\mathrm{zu}$ fachsprachlichen Texten Zugang haben. Unterschiedliche Ausprägungen können die Wahrnehmung oder das Verstehen beeinträchtigen. Barrieren können auch in Kombination auftreten. Eine Sinnesbarriere etwa führt dazu, dass Menschen, die blind oder taub sind, eine Mitteilung nicht wahrnehmen können, weil der jeweilige Sinn nicht funktionsfähig ist (vgl. Schubert 2016: 18).

1 Übersetzen ist damit nicht gemeint. Denn die deutsche Sprache bleibt bestehen. Anders Bredel/Maaß (2016a: 181), die sogar eine Verbindung zur „kontrastiven Linguistik“ ziehen.
Gemäß dem Behindertengleichstellungsgesetz (§ 1 Abs. $1 \mathrm{~S} .1 \mathrm{BGG})^{2}$ sind Leichte Sprache-Texte in erster Linie an Menschen mit Behinderung adressiert. Erklärtes Ziel ist,„,die Benachteiligung von Menschen mit Behinderungen zu beseitigen und zu verhindern sowie ihre gleichberechtigte Teilhabe am Leben in der Gesellschaft zu gewährleisten und ihnen eine selbstbestimmte Lebensführung $\mathrm{zu}$ ermöglichen." Damit wird erstmals gesetzlich verankert, dass Menschen, die „langfristige körperliche, seelische, geistige oder Sinnesbeeinträchtigungen haben“, das gleiche Anrecht haben wie Menschen ohne Behinderungen, ihr Leben selbstbestimmt zu führen (§ 3 S. 1 BGG).

Ein Blick in verschiedene Regelwerke (Netzwerk Leichte Sprache ${ }^{3}$, BITV 2.0, Inclusion Europe) gibt Aufschluss über die von der Praxis genannten Adressaten. Im engeren Sinn richten sich Leichte Sprache-Texte an Menschen mit kognitiven und sensorischen Beeinträchtigungen, die Lernschwierigkeiten haben. Inkludiert sind Personen mit zerebralen Störungen, Demenz, Aphasiker und prälingual Gehörlose. Die größte Zielgruppe bilden die vom funktionalen Analphabetismus Betroffenen. Im weiten Sinn zählen auch Migranten dazu, und diejenigen, die Deutsch als Zweitsprache oder als Fremdsprache erlernen. Die Botschaft der Regelwerke ist, dass ein sehr großer Adressatenkreis aus Leichter Sprache Nutzen ziehen kann. Diese Meinung teilt die Übersetzungswissenschaftlerin Christiane Maaß (2015: 15) und empfiehlt allen Menschen, die mit ausgangssprachlichen Standard- und Fachtexten nicht zurechtkommen, ,in der konkreten Situation lieber die ,leichtere“Version (...) als Zusatzangebot“ zu verwenden.

\section{Die Heterogenität}

Linguistisch betrachtet ist die heterogene breite Adressatenschaft problematisch. Die oberste Maxime der Leichten Sprache ist Verständlichkeit. Textverständlichkeit ist aber keine Eigenschaft per se. Vielmehr geht es um eine Beziehungsfunktion, die das Vorwissen des Rezipienten ebenso einschließt wie Motivation, Text- und Sprachkompetenz. Text- und Leserfaktoren interagieren. Verstehen hängt

2 Das BGG ist im Jahr 2002 in Kraft getreten und zuletzt im Jahr 2016 geändert worden. Es regelt die Belange von Personen mit Behinderung für Bundesbehörden, wurde aber in die Landesgesetzgebung überführt, sodass daneben separate Landesgleichstellungsgesetze existieren.

3 Abrufbar unter http://www.leichtesprache.org. Die Regeln hat das Bundesministerium für Arbeit und Soziales 2013 gleichlautend in einer eigenen Broschüre übernommen; abrufbar unter http://www.gemein sam-einfach-machen.de. 
nicht allein von Textmerkmalen ab, sondern vom Leser selbst: „Verständlich ist ein Text, wenn seine Adressaten ihn verstehen können“ (Hoffmann 1992: 124). Zweck eines Normtextes etwa ist, kommunikativ $\mathrm{zu}$ wirken, sodass Adressaten ihn in ihre Handlungsplanung einbeziehen können.

Das Handbuch der Rechtsförmlichkeit vom Bundesjustizministerium, das die Fachministerien bei der Spracharbeit leitet, äußert sich zur Adressatenfrage. Für uns ist interessant, dass das Ministerium von der pauschalen Forderung abgerückt ist, dass Vorschriften „für alle“ verständlich zu sein haben (vgl. Luttermann 2010 b: 149). Stattdessen unterscheidet es zwischen einer „unbegrenzten“ und „eingeschränkten“ Zielgruppe. Danach gibt es Gesetze nur für spezielle Fachleute und Gesetze für Experten und Laien zusammen. Die mehrfachadressierten Texte wenden sich neben Juristen an eine „durchschnittlich verständig(e) Person“ (BMJ 2008: 55). Der Laie ist in diesem Sinne der informierte Durchschnittsbürger (ein ,vernünftiger und aufmerksamer Mensch“; Luttermann 2010a: 147). Die Adressatenschaft erschließt sich hier intersubjektiv über das Merkmal Wissensstand.

Dagegen erscheint die Zielgruppe der Leichten Sprache praktisch willkürlich und inkongruent. Während funktionale Analphabeten das sinnentnehmende Lesen kaum beherrschen, verfügen viele Migranten über eine Lesekompetenz in der Erstsprache. Nicht jeder Laie mit einer Behinderung braucht Leichte Sprache. Was adressatenangemessen ist, gehört stärker in den Fokus der wissenschaftlichen Betrachtung gerückt. Jedenfalls ist Heterogenität für das Verständlich-Machen von Rechtstexten zu vermeiden. Vielmehr ist der Laie nach Möglichkeit jeweils dort abzuholen, wo er wissens- und bedarfsmäßig steht. Die Sprachwissenschaft kann den Wissenstransfer für selbstbestimmte Anschlusshandlungen von Laien befördern (Kap. VI, VII).

\section{Genese der Leichten Sprache}

\section{Einbettung}

Leichte Sprache wurde vorrangig intuitiv in der Praxis entwickelt. Der Beginn liegt in Amerika, wo bereits in den 1970er Jahren die Gruppe People First von Menschen mit Lernschwierigkeiten gegründet wurde. Im Jahr 1988 folgte Inclusion Europe, eine europäische Vereinigung von Menschen mit geistiger Behinderung und ihrer Familien. Zweck ist, wie auch in Amerika, die weitgehende Autonomie von Menschen mit Beeinträchtigungen in verschiedenen Lebenslagen zu ermöglichen. In Deutschland hat
Leichte Sprache durch die Ratifizierung der UN-Behindertenrechtskonvention im Jahr 2009 einen Aufschwung genommen. Artikel 2 UN-BRK listet konkret Aspekte von Kommunikation auf:

\footnotetext{
„Im Sinne dieses Übereinkommens schließt „Kommunikation“ Sprachen, Textdarstellung, Brailleschrift, taktile Kommunikation, Großdruck, leicht zugängliches Multimedia sowie schriftliche, auditive, in einfache Sprache übersetzte, durch Vorleser zugänglich gemachte sowie ergänzende und alternative Formen, Mittel und Formate der Kommunikation, einschließlich leicht zugänglicher Informations- und Kommunikationstechnologie, ein“.
}

Als solche werden „leichte Zugänglichkeit zur Informations- und Kommunikationstechnologie“ und „einfache Sprache“ genannt. Es fällt auf, dass die UN-Konvention, die den Grundstein für den Ausbau von Leichter Sprache in der deutschen Gesetzgebung legt, selbst nicht von leichter Sprache spricht. Erstmals explizit fixiert die Barrierefreie-Informationstechnik-Verordnung von 2011 „Leichte Sprache" für den Transfer von Behördenkommunikation. ${ }^{4}$ Die Verordnung (§ 3 Abs. 2S. 1 BITV 2.0) legt fest, dass neu gestaltete Webauftritte des Bundes ,in Deutscher Gebärdensprache und in Leichter Sprache bereitzustellen“ sind.

\section{Die Zielgruppenprüfung}

Die Bestimmungen der BIT-Verordnung sind in sprachwissenschaftlicher Hinsicht wenig hilfreich. Recht vollständig aus den sprachlichen Ressourcen des Alltags heraus zu formulieren, ist legislativ und administrativ nicht umsetzbar. Die Terminologisierung erschwert verständliche Kommunikation, weil den gebräuchlichen Wörtern ein neuer Bedeutungsgehalt zugeordnet wird, ohne dass sich aber Graphie und Phonetik ändern, wie am Beispiel der Leihe veranschaulicht (Kap. III). Eine Zielgruppenprüfung für die Präsentation von Informationen in Leichter Sprache im Internet oder Intranet ist nicht vorgesehen. Die Prüfung ist aber notwendig, um das tatsächliche Fachwissen zu ermessen. Es geht nicht darum, die Texte auf Regelkonformität zu prüfen (Leichte Sprache-Texte basieren auf einem umfangreichen, aber uneinheitlichen Kriterienkatalog), ${ }^{5}$ sondern es geht um reales Verstehen (Konstruktion von Sinn) und Vorerfahrungen der Probanden.

Die Angewandte Linguistik untersucht schon empirisch die tatsächlichen und ermittelbaren Grundlagen des

4 Angelehnt an die Web Content Accessibility Guidelines (WCAG); siehe Bredel/Maaß (2016b: 19).

5 Siehe Gross (2015: 95); Schädler/Reichstein (2015: 46f.). 
Verstehens von Laien (Medizin, Politik, Recht, Verwaltung, Technik). Hier setzt auch mein Rechtslinguistisches Verständlichkeitsmodell an, das ich für die Optimierung von Fachkommunikation zwischen Experten und Laien entwickelt habe. Auszuloten ist, inwieweit es für die Adressaten der klaren Sprache trägt.

\section{Rechtslinguistisches Verständlichkeitsmodell}

\section{Ziel und Ansatz}

Das Rechtslinguistische Verständlichkeitsmodell (RVM) zielt darauf, für Lexeme, die in der Rechts- und Gemeinsprache vorkommen, Gemeinsamkeiten und Problemlagen zu ermitteln, die sich aus den Bedeutungsgebungen entwickeln. Es gibt Laien als Textadressaten ein stärkeres analytisches Gewicht. Das Modell setzt die Erkenntnis der Fachsprachenforschung um, für eine verständliche Darstellung von Fachwissen neben dem Expertenwissen auch die Eigenperspektive der Laien zu beachten (vgl. Luttermann 2001: 154; 2002: 98 ff.). Kennzeichen ist eine präzise Abfolge von vier Analyseschritten, um zunächst je Expertenwissen (Theoriemuster) und Laienwissen (Empirie- und Ergebnismuster) durch Befragungen (Interview, Fragebögen) zu eruieren und schließlich die semantischen Bedeutungskonnotationen für Schnittmengen und Unterschiede aufeinander zu beziehen (Vergleichsmuster).

Erst im Spektrum der Verwendung werden die Bedeutungen eines Ausdrucks und die zugrunde liegenden Wissensstrukturen fassbar. Der mehrperspektivische Ansatz gewichtet Möglichkeiten und Grenzen für Optimierungsprozesse in der Rechts- und Fachkommunikation. Das Theoriemuster zeigt die fachsprachliche Gebrauchssemantik aus Expertensicht (Gesetzgeber, Rechtsprechung, Kommentarliteratur, Lehrbücher). Das Empiriemuster legt offen, was ein Rechtslaie versteht, und das Ergebnismuster, was viele Laien begreifen. Das Vergleichsmuster schließlich vergleicht die fach- und gemeinsemantischen Lesarten auf Gemeinsamkeiten und Unterschiede.
Im Strafrecht (Diebstahl § 242 StGB) bereitet zum Beispiel das Wort Sache Verstehensprobleme. Denn anders als Laien interpretieren Juristen Tiere als Sache. Die Tötung eines Hundes stellt eine Sachbeschädigung dar (vgl. Luttermann 2010 a: 148). Mit finden für Finderlohn (§ 971 BGB) meinen Juristen eine Geschäftsbesorgung, d.h. der Finder nimmt eine verlorene Sache in Besitz, während Laien „wahrnehmen“ und „entdecken“ darunter verstehen. Für sie ist der Sehsinn bedeutungsbestimmend (vgl. Luttermann 2015: 284). Die Bedeutungsunterschiede spiegeln, wo die Kommunikation zwischen dem Gesetzgeber und den Adressaten nicht gut funktioniert.

\section{Optimierungsprozess}

Am Sprachgebrauch zeigt sich, was und wie viel Laien jeweils verstehen. Durch die empirische Offenlegung der Bedeutungskonzepte können die semantisch relevanten Wissenselemente der Wörter rekonstruiert und miteinander verglichen werden. Zugleich wird deutlich, dass die Mehrperspektivität und Beteiligung der Wissenschaft an der Spracharbeit für die Rechtsetzung förderlich sind. Im rechtslinguistischen Optimierungsprozess geht es um pragmatische Angemessenheit. Juristen und Linguisten suchen Lösungen für eine angenäherte Verständigungsebene zwischen Experten- und Laienwissen. Dabei begrenzt der Empfängerhorizont sach- und adressatenangemessen, was rechtlich machbar und für den Adressatenkreis zu verstehen ist. Das ist für die Rechtssicherheit notwendig.

Ein mehrschrittiges Verfahren, das Sachkompetenz und Verstehensleistungen komplementär behandelt, ist auch für Texte in klarer Sprache anzustreben. Denn Experten und Laien sind heuristisch gleichermaßen wichtig.

\section{Textfunktion beibehalten}

Durch Perspektivenvergleich können juristische Texte adressatenspezifisch aufbereitet und Differenzen minimiert (idealerweise sogar überbrückt) werden (Kap. V.2, VI.1). Die Forschungsstelle Leichte Sprache in Hildesheim

\begin{tabular}{|lll|}
\hline Theoriemuster & $\rightarrow$ & Expertenperspektive \\
Empiriemuster & $\rightarrow$ & Perspektive eines Laien \\
Ergebnismuster & $\rightarrow$ & Perspektive vieler Laien \\
Vergleichsmuster & $\rightarrow$ & Experten- und Laienperspektive \\
\hline
\end{tabular}

Abbildung 1: Analyseschritte (RVM). 
schließt umformulierte Rechtstexte von jeglicher Haftung aus, d.h. die veränderten Texte sind nicht rechtsgültig und justiziabel (vgl. Bredel/Maaß 2016b: 26f.). Damit wird sie ihrem Anspruch nicht gerecht, die „Textfunktion stabil“ (Maaß 2015: 141f.) zu halten. Denn Appelltexte werden in reine Informationstexte umgewandelt. Texte im Kommunikationsbereich Recht erfüllen aber nicht vorrangig die Funktion der Informationsvermittlung. Mit der Umwandlung verlieren die Texte nicht nur ihren spezifischen appellativen Charakter. Es verschiebt sich vielmehr die Zielsetzung. Statt der Arbeit an der Verständlichkeit bzw. Optimierung von Gesetzen ist das Ziel der Anpassung, Texte in Leichter Sprache über Gesetze zu erarbeiten, also über einen Rechtsgegenstand zu informieren.

Für diese kommunikative Aufgabe ist ein funktionaler Wechsel der Textsorte angemessen und nachvollziehbar. Für meinen Laienbegriff und seine Einbettung in die Fachkommunikation ist die Spezifik der Textsorte dagegen nicht verhandelbar. Die verschiedenen Textsorten werden nicht allesamt zu informierenden Texten neutralisiert. Den Laien wird zugetraut und zugemutet, sich mit dem juristischen Stil und Sprachregister auseinanderzusetzen. Sie sollen aktiv ein Textsortenwissen aufbauen und erweitern können. Eine Textsorte gibt Aufschluss darüber, was ein Text bezweckt. Spracherfahrung und Textsortenkonvention vorzuenthalten und individuelle Kompetenzen zu unterschätzen, halte ich für eine gelebte Teilhabe für wenig zielführend.

\section{Begriffliche Einordnung der klaren Sprache}

\section{Semantische Konkurrenzen}

Die Leichte Sprache gilt als Varietät der deutschen Sprache und wird als ein festgefügtes wirksames Konzept für Barrierefreiheit und Inklusion wahrgenommen, weil sie Komplexität reduziert. Die Reduktion betrifft primär Syntax, Lexik und Inhalt (vgl. Maaß 2015: 11 f.), wobei eine empirische Überprüfung der Wirksamkeit der aufgestellten Restriktionen fehlt. Definiert wird Leichte Sprache primär über bestimmte sprachliche und typographische Regeln, die eine Vermittlungsfunktion für die Textverständlichkeit haben. Die Regeln der verschiedenen Regelwerke sind zum Teil ebenso widersprüchlich wie es Bezeichnungen für das Praxis-Phänomen Leichte Sprache gibt (vgl. Lange/Bock 2016: 120). Ausdrücke wie leicht lesbar, Leicht Lesen, leicht gesagt, einfach gesagt, verständlich gesagt, einfache Sprache werden für dieselbe Zielgruppe gebraucht.
Die Semantik der Einstufungsausdrücke ist vornehmlich politisch und ökonomisch motiviert und ein Versuch strategischer Begriffsbildung. Worin die Unterschiede tatsächlich bestehen, bleibt vage. Ungeklärt ist aufgrund der mangelnden Forschung vor allem, ob die Abgrenzung der leichten von der einfachen Sprache linguistisch überhaupt trägt. Auffällig ist jedenfalls, dass die UN-Behindertenrechtskonvention in den Sprachfassungen dt. „einfache Sprache“, engl. „plain-language“, frzs. „langue simplifiée“ und it. „linguaggio semplice“ verwendet (Art. 2 UN-BRK), was man mit > vereinfachter Sprache< übersetzen kann. Im Kern geht es also um Vereinfachung (und nicht um LeichtMachen). „Leichte Sprache“ ist negativ konnotiert und hat das Stigma der Simplifizierung, die Adressaten unterfordern kann (vgl. Bock 2014: 34). ${ }^{6}$ - Besinnen wir uns auf sprachwissenschaftliche Grundlagen für die Kommunikation.

\section{Konversationsmaximen}

Kommunikative Interaktion basiert mit Herbert Grice auf Kooperation. Von den Interaktanten wird erwartet, dass sie kooperativ und rational handeln, damit Verständigung gelingt. Das allgemeine Kommunikationsprinzip geht vom kommunikativen Zweck aus: „Mache deinen Gesprächsbeitrag jeweils so, wie es von dem akzeptierten Zweck oder der akzeptierten Richtung des Gesprächs, an dem du teilnimmst, gerade verlangt wird“ (Grice 1979: 248). Für die praktische Umsetzung der Kommunikationsregel formuliert Grice die Konversationskategorien Quantität, Qualität, Relation und Modalität. Den vier Kategorien ordnet er jeweils Konversationsmaximen zu. Die Maximen sind keine Vorschriften, sondern generelle Erwartungen, die Interaktanten im Kommunikationsprozess voneinander ausbilden, um Äußerungen zu interpretieren. Verletzte Maximen werden uminterpretiert, sodass die Annahme der Kooperativität wieder möglich ist.

Die Quantitätsmaxime stellt auf die Informationsmenge ab (Unter- und Obergrenze). Die Beiträge sollen so informativ wie nötig und nicht informativer als nötig sein. Information setzt allerdings Verständlichkeit voraus. Qualität rekurriert auf den Wahrheitsgehalt einer Aussage und Relation auf die Relevanz hinsichtlich Thema und Situation. Die Maxime der Modalität regelt die Art und Weise, wie

6 Leichte Sprache-Texte gelten als unterkomplex, wenig herausfordernd, zu leicht. Die Kritik entzündet sich primär am Binnenstrich, redundanten Satzbau, Passivverbot und an infantilisierenden Bildern. Der restringierte Code entspricht nicht dem normativen Bildungs- und Sprachideal. 
etwas gesagt wird und fordert Klarheit durch Disambiguierung („eindeutig machen“) und Gliederung (logische Reihenfolge). Die Aufforderung „Sei klar.“ weist uns die Richtung für die Zugänglichkeit bzw. Optimierung klarer Texte in der Experten-Laien-Kommunikation.

\begin{tabular}{|l|l|}
\hline Konversationskategorie & Konversationsmaxime \\
\hline Quantität & $\begin{array}{l}\text { 1. Mache deinen Beitrag für den Zweck } \\
\text { des Gesprächs so informativ wie nötig. } \\
\text { 2. Mache deinen Beitrag nicht } \\
\text { informativer als nötig. }\end{array}$ \\
\hline Qualität & $\begin{array}{l}\text { Versuche deinen Beitrag so zu machen, } \\
\text { dass er wahr ist. } \\
\text { a) Sage nichts, was du für falsch hältst. } \\
\text { b) Sage nichts, wofür dir hinreichende } \\
\text { Gründe fehlen. }\end{array}$ \\
\hline Relation & $\begin{array}{l}\text { Sei relevant in Bezug auf den } \\
\text { Gesprächsgegenstand und die } \\
\text { Gesprächssituation. }\end{array}$ \\
\hline Modalität & $\begin{array}{l}\text { Sei klar. } \\
\text { a) Vermeide Unklarheit. } \\
\text { b) Vermeide Mehrdeutigkeit. } \\
\text { c) Vermeide unnötige Weitschweifigkeit. } \\
\text { d) Vermeide Ungeordnetheit. }\end{array}$ \\
\hline
\end{tabular}

Abbildung 2: Konversationsmaximen.

\section{Klarheit}

Klare Sprache als Kommunikationsmittel zielt auf eine adäquate verständliche Kommunikationsvermittlung für Laien. ${ }^{7}$ Es handelt sich dabei um eine funktionale Sprachgebrauchsform des Deutschen. Das Wort „klar“ stammt von lt. clarus (>hell, deutlich $<$ ) und meint >klarmachen, deutlich machen, verständlich machen<. Im Fokus stehen die sprachlichen Ausdrucksmöglichkeiten, der inhaltliche Gehalt und das Weltwissen der Adressaten. Klarheit umfasst damit zwei Komponenten, die für unsere Zwecke bedeutsam sind: Zum einen stellt das Attribut auf die Erfordernisse des Textinhalts ab, d.h. auf Deutlichkeit bzw. Verdeutlichung des Gesprächsgegenstandes, indem etwa die thematische Entfaltung (deskriptiv, argumentativ, explikativ, narrativ) und Kohärenz untersucht werden.

Zum zweiten ist Verständlichkeit in dem Sinn inhärent, dass Textinhalte für jemanden verfasst sind. In der

7 Das niederländische Parlament hatte 1973 eine Kommission für klare Sprache (Commissie Duidelijke Taal) ernannt. In Norwegen wurde 1990 die Leicht Lesen-Zeitung Klar Tale (klare Stimme) gegründet. Nach Leskelä (2015: 169) bedeutet selko im Finnischen „Klarheit und Verständlichkeit“.
Experten-Laien-Kommunikation richten sich die Inhalte mit der vorgeschlagenen Perspektivenerweiterung an Laien unterschiedlicher Bedürfnisse und Niveaus. Für den Klarheitsbegriff ist konstitutiv, die Ausdifferenzierung der textuellen Niveaustufen nicht am Gemeinsamen Europäischen Referenzrahmen für Lerner von fremden Sprachen auszurichten, der die Sprachkompetenz in sechs Schwierigkeitsstufen (A1, A2, B1, B2, C1, C2) festlegt. Darauf basiert das Capito-Stufenmodell. ${ }^{8}$ Ausschlaggebend für die Textgraduierung für Menschen mit Behinderung ist vielmehr das Hintergrundwissen der Rezipienten selbst, das empirisch durch Befragung erst zu ermitteln ist (Kap. VI.1).

Ich ziehe die Bezeichnung „klare Sprache“ der Bezeichnung „Leichte Sprache“ vor, weil die Sach- und Adressatenangemessenheit, auf die es bei fachlicher Kommunikationsoptimierung entscheidend ankommt, enthalten sind. Texte lassen sich sinnerhaltend (ohne den Inhalt des Ausgangstextes zu tangieren) nicht beliebig umformulieren, wie Psycholinguisten behaupten (Luttermann 2002: 50; Luttermann/Schäble 2016: 402). Die Kategorie der Sachangemessenheit hat die kommunikative Funktion, Inhaltskonstanz zu wahren. In mehreren Schritten kann unter Berücksichtigung der sprachlichen und inhaltlichen Verstehensleistungen der Probanden ein neuer Text entstehen, ohne dass dabei aber die Textsortenfunktion verloren geht (Kap. VI.3).

Außerdem hat „klare Sprache“ den Vorteil, nicht zu stigmatisieren (,leicht“ steht antithetisch zu „schwer“) und Gefahr zu laufen, selbst eine Sprachbarriere aufzubauen zwischen jenen, die einen vereinfachten Textzugang nutzen müssen (also „leichte“ Texte brauchen), und jenen, die darauf nicht angewiesen sind (und mit „schweren“ Texten zurechtkommen). Der im Zusammenhang der klaren Sprache gebrauchte Laienbegriff wertet nicht. Vielmehr konstatiert er ,wertfrei das Vorliegen bestimmter Wissensniveaus und leitet aus diesen Feststellungen keine positiven oder negativen Urteile über die in Rede stehenden Personen ab“ (Wichter 1994: 55). Beispielsweise benötigt jeder Nichtjurist (unabhängig von einer Behinderung) Vermittlung und Vereinfachung, wenn er gesetzliche Regelungen, die ihn betreffen, genau im Handlungskontext verstehen will (vgl. Bock/Lange 2015: 69). Wir alle sind aktive und informationssuchende Laien auf bestimmten Gebieten und wollen in unserer persönlichen Textrezeption und auf unserem individuellen Weg der Kompetenzaneignung bestmöglich unterstützt werden.

Umzusetzen ist die Vermittlungsaufgabe - also das Zugänglichmachen von fachsprachlichen Texten - durch

8 Siehe Fröhlich/Candussi (2015: 32). 
klare Sprache nach Maßgabe des mehrperspektivischen Ansatzes, der für die Experten-Laien-Kommunikation im Recht schon angewandt wird (Kap. VI). Das Klarheitsgebot vermeidet eine starre Niveaufestlegung, weil die klare Sprache - anders als Leichte Sprache - eben kein Hilfsmittel ist, das ein Sprachsystem allein für Menschen mit Behinderung konstruiert und damit deren Benachteiligung merkmalsspezifisch und regelhaft festschreibt. Vielmehr handelt es sich bei der klaren Sprache um Varietäten des Sprachgebrauchs, gerichtet auf Angemessenheit hinsichtlich Adressaten und Inhalt.

\section{Beispiel: Ordentliche Gerichtsbarkeit}

\section{Klar machen}

Betrachten wir den rechtssprachlichen Begriff „Ordentliche Gerichtsbarkeit“, den Christiane Maaß (2015: 8) folgendermaßen erleichtert: ${ }^{9}$

\section{Ordentliche Gerichtsbarkeit}

2 Personen streiten sich.

Die Personen gehen zu einem Gericht.

Die Personen gehen zu einem ordentlichen Gericht.

Ordentlich hat hier nichts mit sauber zu tun.

Ordentliche Gerichte sind die streitigen Gerichte.

Das Wort streitig kommt von Streit.

Die ordentlichen Gerichte regeln Streite.

Zu den ordentlichen Gerichten gehören zum Beispiel:

- Amts·gerichte.

- Und Land.gerichte.

Aus meiner Sicht sind zugunsten der Klarheit mindestens vier Sätze zu streichen. Der umformulierte Text lautet also:

\section{Ordentliche Gerichtsbarkeit}

2 Personen streiten sich.

Die Personen gehen zu einem ordentlichen Gericht.

Die ordentlichen Gerichte regeln Streite.

Zu den ordentlichen Gerichten gehören zum Beispiel:

- Amts·gerichte.

- Und Land·gerichte.

9 Im Ausgangstext erklärt das niedersächsische Ministerium der Justiz den Fachbegriff unter „Punkt Justiz verstehen“; abrufbar unter www.mj.niedersachsen.de.
Das Netzwerk Leichte Sprache empfiehlt die Konjunktion und am Satzanfang. Allerdings erscheint mir hier der Gebrauch von „und“ (vor „Land-gerichte“) entbehrlich. Außerdem ist der Satz „Zu den ordentlichen Gerichten gehören zum Beispiel“" paraphrasierbar (Stattdessen kann man klar sagen: „Die ordentlichen Gerichte sind zum Beispiel“.) und der Ausdruck „streitige Gerichte“ missverständlich. „Streitig“ bedeutet gemeinsprachlich >umstritten< in der Wendung >jemandem etwas streitig machen $<$ oder $>$ jemandem das Anrecht auf etwas bestreiten< (Dudenredaktion 2014: 828). Dass die ordentlichen Gerichte umstrittene Gerichte sind, ist hier aber nicht gemeint. Das Wort „streitig“ tritt uns als Fachbegriff entgegen, der sich intuitivem Verstehen entzieht und von der nichtstreitigen (freiwilligen) Gerichtsbarkeit abgrenzt, die außer Gerichte auch Notare und Behörden etwa in der Rechtspflege ausüben. Ordentliche Gerichte befassen sich mit zivil- und strafrechtlichen Streitigkeiten.

\section{Weitere Auffälligkeiten}

Das Beispiel zeigt weitere Auffälligkeiten: So ist die Kardinalzahl „zwei“ eine Ziffer und kein Zahlwort. Das Mittel der Kohäsion, die den strukturell-grammatischen Zusammenhang des Textes bildet, ist die Rekurrenz. Das mehrfache Auftreten desselben Lexems ist für die Rechtssprache zwar typisch, um für Eindeutigkeit zu sorgen (vgl. Luttermann 2016: 168f.). Hier wird das Lexem „Gericht“ jedoch gleich neun Mal gebraucht. Es kommen keine Pro-Formen vor. Zum Beispiel wird das Nomen „Personen“ nicht anaphorisch durch das Personalpronomen „sie“ ersetzt. Die Sätze sind kurz und haben nicht mehr als acht Wörter. Sätze ohne Nebensatz und mit begrenzter Wortanzahl heißen „Einfachsatz“ (Brandt 1991: 345f.). Ein Einfachsatz ist aber im Recht nicht automatisch Garant für Verständlichkeit.

Dass kurz nicht gleich einfach impliziert, lässt sich gut am Mord-Paragraphen (§ 211 StGB) illustrieren. Im Gesetz steht der Satz: „Der Mörder wird mit lebenslanger Freiheitsstrafe bestraft.“ Man kann die Rechtsfolge „lebenslange Freiheitsstrafe" nicht so umformulieren, dass der Mörder sein ganzes Leben ins Gefängnis muss, weil rechtlich anderes gemeint ist (Luttermann2016: $177 \mathrm{ff}$.). Der juristische Wissensrahmen evoziert einen hohen Komplexitätsgrad, der für Nichtjuristen kommunikative Barrieren errichtet. Außerdem fällt auf, dass das Konzept „ordentliche Gerichtsbarkeit“ durch konkrete Beispiele erläutert wird. Durch das selektive Herausgreifen findet zugleich eine Bedeutungsreduktion statt. Die rechtssemantischen Ausdrücke („Amts-gerichte, Land.gerichte“) sind voraussetzungsreich und bedürfen ebenfalls einer Erläuterung. 
Überdies steht jeder Satz auf einer neuen Zeile und ist der Satzbau redundant („Die Personen gehen zu einem Gericht.“ / „Die Personen gehen zu einem ordentlichen Gericht.“). Gearbeitet wird mit Einrückungen und bullet points für die Aufzählung. Zusammengesetzte Nomen sind mit einem Punkt geteilt. Die Hildesheimer Forschungsstelle Leichte Sprache argumentiert damit, dass der Mediopunkt leseerleichternd (,reine Lesehilfe“; Maaß 2015: 91) ist, nicht in das grammatische System der deutschen Sprache eingreift und keine Rechtschreibregeln verletzt. Inwiefern der trennende Punkt zwischen Komposita und Affixen $\mathrm{zu}$ einem besseren Verständnis des Textinhalts führt, ist aber noch eine offene Frage und bedarf der Überprüfungen an Probanden. Dagegen empfehlen Leichte Sprache-Regelwerke und der Ratgeber vom Bundesministerium für Arbeit und Soziales bei komplexen Komposita gerade den Bindestrich (Kap. IV.1).

Inwieweit der Text zur ordentlichen Gerichtsbarkeit also klar ist, ist noch mehrschrittig und unter Beachtung der Textsortenkonventionen zu prüfen. Eines macht dieses Beispiel allerdings schon deutlich: Letztlich hängt Kommunikation an der Sach- und Adressatenangemessenheit, also am Empfängerhorizont, der interdisziplinär zu gestalten ist (Kap. VII.3).

\section{Schluss}

Der vorliegende Beitrag steht in dem Bemühen, das Praxisphänomen Leichte Sprache durch linguistisches Grundlagenwissen $\mathrm{zu}$ theoretisieren. Denn es existieren bisher kaum Bezüge zu theoretischen Ansätzen der Sprachwissenschaft. ${ }^{10}$ Ich habe mich hier auf die Textlinguistik, Fachkommunikation und Rechtslinguistik beschränkt. Mein besonderes Anliegen ist, Anforderungen der externen Fachkommunikation in Leichter Sprache in den Forschungsstand der erweiterten Experten-Laien-Kommunikation zu überführen. Die Einbeziehung der bisher nicht eingebundenen Adressaten habe ich mit einer neuen Begrifflichkeit verknüpft: „Klare Sprache“ (Kap. VII) und beispielhaft veranschaulicht. Der Begriff „klare Sprache“ erweist sich als Scharnierstelle zwischen Experten und Laien, weil er am Empfängerhorizont ausgerichtet ist und Adressaten nicht abwertet. Mit „leicht“ ist mitnichten das Sprachsystem, sondern eine bestimmte Personengruppe gemeint. Klarheit gilt dagegen für jedweden Text und für jedermann.

10 Fix (2017: 165ff.) setzt textlinguistisch an.
Mit den Laienzielgruppen ist noch viel Forschung nötig, um funktionale Vermittlungsvarietäten für Textverständlichkeit in klarer Sprache zu ermitteln. Einen möglichen Weg habe ich mit dem Ansatz der Mehrperspektivität und meinem Verständlichkeitsmodell aufgezeigt (Kap. VI). Das Modell basiert nicht auf Einschätzungen (Schätzurteile), sondern ergründet dezidiert in vier Analyseschritten das Konzept- und Sprachwissen der Adressaten. Die empirischen Befunde gehen in die Textarbeit mit der klaren Sprache ein, die sich elementar von der reduktiven Leichte-Sprache-Arbeit abhebt. Kommunikationsbereichsspezifische Analysen können Möglichkeiten und Grenzen ausloten, das Laienkontinuum sprachlich und inhaltlich auszudifferenzieren.

\section{Literatur}

Adamzik, Kirsten (2016): Textlinguistik. Grundlagen, Kontroversen, Perspektiven. 2. Auflage. Berlin: de Gruyter.

Bock, Bettina (2014): „Leichte Sprache“: Abgrenzung, Beschreibung und Problemstellungen aus Sicht der Linguistik. In: Jekat, Susanne et al. (Hrsg.): Sprache barrierefrei gestalten. Perspektiven aus der Angewandten Linguistik. Berlin: Frank und Timme, 17-51.

Bock, Bettina/Lange, Daisy (2015): Was ist eigentlich „Leichte Sprache"? Der Blick der Sprachwissenschaft. In: Candussi, Klaus/ Fröhlich, Walburga (Hrsg.): Leicht Lesen. Der Schlüssel zur Welt. Wien: Böhlau, 63-79.

Brandt, Wolfgang: Müssen Gesetze schwer verständlich sein? Einwände eines Linguisten gegen Schutzbehauptungen der Juristen. In: Eckert, Jörn/Hattenhauer, Hans (Hrsg.) (1991): Sprache - Recht - Geschichte. Rechtshistorisches Kolloquium 5.-9. Juni 1990 an der Christian-Albrechts-Universität zu Kiel. Heidelberg: Müller, 339-361.

Bredel, Ursula/Maaß, Christiane (2016a): Leichte Sprache. Theoretische Grundlagen. Orientierung für die Praxis. Berlin: Dudenverlag.

Bredel, Ursula/Maaß, Christiane (2016b): Ratgeber Leichte Sprache. Die wichtigsten Regeln und Empfehlungen für die Praxis. Berlin: Dudenverlag.

Brinker, Klaus (2005): Linguistische Textanalyse. Eine Einführung in Grundbegriffe und Methoden. 6. Auflage. Berlin: Schmidt.

Bundesministerium der Justiz (Hrsg.) (2008): Handbuch der Rechtsförmlichkeit. Empfehlungen des Bundesministeriums der Justiz für die rechtsförmliche Gestaltung von Gesetzen und Rechtsverordnungen nach § 42 Absatz 4 und $\S 62$ Absatz 2 der Gemeinsamen Geschäftsordnung der Bundesministerien. 3. Auflage. Köln: Bundesanzeiger.

Dudenredaktion (Hrsg.) (2014): Das Herkunftswörterbuch. Etymologie der deutschen Sprache. 5. Auflage. Berlin: Dudenverlag.

Fix, Ulla (2017): „Schwere“ Texte in „Leichter Sprache“ - Voraussetzungen, Möglichkeiten und Grenzen (?) aus textlinguistischer Sicht. In: Bock, Bettina/Fix, Ulla/Lange, Daisy (Hrsg.): ,Leichte Sprache“ im Spiegel theoretischer und angewandter Forschung. Berlin: Frank und Timme, 163-188. 
Fröhlich, Walburga/Candussi, Klaus (2015): Informationsbarrieren und Wege zu ihrer Überwindung. Das Konzept „Barrierefreie Information“, seine Herleitung und seine Funktionen. In: Candussi, Klaus/Fröhlich, Walburga (Hrsg.): Leicht Lesen. Der Schlüssel zur Welt. Wien: Böhlau, 9-38.

Grice, Herbert (1979): Logik und Konversation. In: Meggle, Georg (Hrsg.): Handlung, Kommunikation, Bedeutung. Frankfurt am Main: Suhrkamp, 243-265.

Gross, Susanne (2015): Regeln und Standards für leicht verständliche Sprache. Ein Rundblick. In: Candussi, Klaus/Fröhlich, Walburga (Hrsg.): Leicht Lesen. Der Schlüssel zur Welt. Wien: Böhlau, 81-106.

Hartmann, Peter (1971): Texte als linguistisches Objekt. In: Stempel, Wolf-Dieter (Hrsg.): Beiträge zur Textlinguistik. München: Fink, 9-29.

Hoffmann, Ludger (1992): Wie verständlich können Gesetze sein? In: Grewendorf, Günther (Hrsg.): Rechtskultur als Sprachkultur. Zur forensischen Funktion der Sprachanalyse. Frankfurt am Main, 122-154.

Lange, Daisy/Bock, Bettina (2016): Was heißt „Leichte“ und „einfache Sprache“? Empirische Untersuchungen zu Begriffssemantik und tatsächlicher Gebrauchspraxis. In: Mälzer, Nathalie (Hrsg.): Barrierefreie Kommunikation - Perspektiven aus Theorie und Praxis. Berlin: Frank und Timme, 117-134.

Leskelä, Leealaura (2015): Von Selko zu Leicht Lesen. Ein nordischer Blick auf die praktische Durchsetzung eines Bügerrechtes. In: Candussi, Klaus/Fröhlich, Walburga (Hrsg.): Leicht Lesen. Der Schlüssel zur Welt. Wien: Böhlau, 169-185.

Luttermann, Karin (2001): Empfängerhorizont in der juristischen Experten-Laien-Kommunikation. In: Fachsprache 3-4, 153-159.

Luttermann, Karin (2002): Juristensprache als Kommunikationsaufgabe - Grundlagen und Perspektiven. In: Der Deutschunterricht 5, 46-55.

Luttermann, Karin (2010a): Heutige Wechselwirkungen zwischen Allgemein- und Rechtssprache: Deutsch. In: Fischer, Roswitha (Hrsg.): Sprache und Recht in großen europäischen Sprachen. Juristische Begriffsbildung im Spannungsfeld zwischen Fachsprachlichkeit und allgemeiner Verständlichkeit. Regensburg: Universitätsverlag, 139-154.

Luttermann, Karin (2010b): Verständliche Semantik in schriftlichen Kommunikationsformen. In: Fachsprache 3-4, 145-162.

Luttermann, Karin (2015): Linguistisch-pragmatische Zugänge zur Rechtssemantik: Was gesagt, was gemeint und was verstanden wird. In: Vogel, Friedemann (Hrsg.): Zugänge zur Rechtssemantik. Interdisziplinäre Ansätze im Zeitalter der Mediatisierung. Berlin: de Gruyter, 275-292.
Luttermann, Karin (2016): Indeterminismus und Performanz in der Sprache am Beispiel der Bedeutung von lebenslanger Freiheitsstrafe. In: Bülow, Lars et al. (Hrsg.): Performativität in Sprache und Recht. Berlin: de Gruyter, 163-188.

Luttermann, Karin/Schäble, Pia (2016): Wissenstransfer in der Wirtschaftskommunikation: Zum Sprachverständnis der europäischen Finanzkrise aus Experten- und Laiensicht. In: Kastens, Inga/Busch, Albert (Hrsg.): Handbuch Wirtschaftskommunikation. Interdisziplinäre Zugänge zur Unternehmenskommunikation. Tübingen: Francke, 389-433.

Maaß, Christiane (2015): Leichte Sprache. Das Regelbuch. Berlin: Lit. Schädler, Johannes/Reichstein, Martin (2015): „Leichte Sprache“ und Inklusion. Fragen zu Risiken und Nebenwirkungen. In: Candussi, Klaus/Fröhlich, Walburga (Hrsg.): Leicht Lesen. Der Schlüssel zur Welt. Wien: Böhlau, 39-61.

Schubert, Klaus (2016): Barriereabbau durch optimierte Kommunikationsmittel: Versuch einer Systematisierung. In: Mälzer, Nathalie (Hrsg.): Barrierefreie Kommunikation - Perspektiven aus Theorie und Praxis. Berlin: Frank und Timme, 15-33.

Shannon, Claude/Weaver, Warren (1949): The Mathematical Theory of Communication. Urbana: University of Illinois Press.

Wichter, Sigurd (1994): Experten- und Laienwortschätze. Umriß einer Lexikologie der Vertikalität. Tübingen: Niemeyer.

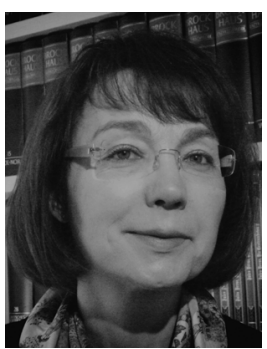

\section{Prof. Dr. Karin Luttermann}

Katholische Universität Eichstätt-Ingolstadt Deutsche Sprachwissenschaft Universitätsallee 1

85072 Eichstätt

karin.luttermann@ku.de

Karin Luttermann, Dr. phil. habil., Studium der Germanistik und Rechtswissenschaften an der Universität Münster. Promotion 1996 über Gesprächsanalyse in der Gerichtskommunikation. Habilitation 2009 über Sprachgebrauch und Verständlichkeit in der Rechtskommunikation. Seit 2015 Professorin für Deutsche Sprachwissenschaft an der Universität Eichstätt-Ingolstadt. Forschungsschwerpunkte: Kommunikation in Recht, Wirtschaft und Werbung, Fachsprachenund Verständlichkeitsforschung, Wissenstransfer, Textlinguistik, Angewandte Linguistik, Orthographie. 